\title{
Evaluation of blood culture systems for detection of the intestinal spirochaete Brachyspira (Serpulina) pilosicoli in human blood
}

\author{
C. JOSEPHINE BROOKE, K. RINI MARGAWANI, ALAYNE K. PEARSON*, THOMAS V. RILEY*†, \\ IAN D. ROBERTSON and DAVID J. HAMPSON
}

Division of Veterinary and Biomedical Sciences, Murdoch University, Murdoch, Western Australia 6150, * Division of Microbiology and Infectious Diseases, Western Australian Centre of Pathology and Medical Research, Nedlands WA 6009 and †Department of Microbiology, University of Western Australia, Nedlands WA 6009, Australia

\begin{abstract}
The anaerobic intestinal spirochaete Brachyspira (Serpulina) pilosicoli has been isolated from the bloodstream of French patients by manual blood culture systems. The purpose of this study was to determine whether the automated and manual blood culture systems used in Australia are suitable for growth and detection of this organism. Strains of $B$. pilosicoli were added to human blood to give concentrations ranging from $1 \times 10^{4}$ to $1 \times 10^{1}$ spirochaetes $/ \mathrm{ml}$ and $10-\mathrm{ml}$ volumes were inoculated into the media. Three strains of $B$. pilosicoli grew slowly in all manual Hémoline and BBL Septi-Chek formulations tested. Subcultures taken between 2 and 10 days after inoculation yielded growth only after incubation for a further 5-8 days. Growth and automated detection were achieved in the BACTEC system with Anaerobic/F medium with or without Fastidious Organism Supplement. Minimum time to signal for nine strains varied between 5.6 and 14.9 days, with a minimum concentration of $10^{1}$ spirochaetes $/ \mathrm{ml}$ of blood being detected. None of nine strains gave a positive signal in the BacT/Alert system when FAN Anaerobic culture bottles were used; however, four strains were detected by subculture taken at 7 or 14 days after inoculation. When Anaerobic medium was used in the BacT/Alert system, two of three strains gave a signal and the other strain grew and was detected by subculture. Spirochaetaemias caused by $B$. pilosicoli may be unrecognised because detection time by the signal or subculture exceeds 5 days.
\end{abstract}

\section{Introduction}

Brachyspira (previously Serpulina) pilosicoli is an anaerobic spirochaete that colonises the large intestine of man and many animal species [1-3]. In pigs, large numbers of these spirochaetes are found attached by one end of their cell to the colonic mucosa, and this colonisation is associated with colitis and diarrhoea $[3,4]$. In man the end-on attachment of spirochaetes is referred to as intestinal spirochaetosis (IS) [1,5]. Colonisation of the large intestine by $B$. pilosicoli apparently occurs quite commonly ( $>30 \%$ prevalence) in man in developing countries [2,6], in Australian Aborigines [7], in homosexual males [5] and in patients with human immunodeficiency virus (HIV) infection

Received 4 Jan. 2000; revised version received 5 Feb. 2000; accepted 5 April 2000.

Corresponding author: Dr D. J. Hampson (e-mail:

hampson@numbat.murdoch.edu.au).
[8]. Clinically, colonisation has been linked to various intestinal disorders, including chronic diarrhoea and rectal bleeding $[1,9,10]$. A volunteer who drank cultures of a human isolate of $B$. pilosicoli became colonised and developed abdominal discomfort and headaches after a prolonged incubation period $(>1$ month) [11]. Invasive colitis and hepatitis associated with intestinal spirochaetes have also been recorded $[12,13]$, although in some cases $B$. aalborgi rather than $B$. pilosicoli may have been involved $[14,15]$.

The potential pathogenicity of $B$. pilosicoli was emphasised recently by its isolation from the bloodstream of seven patients in France and the USA $[16,17]$. The patient in the USA had HIV infection and was receiving chemotherapy for Kaposi's sarcoma. The six French patients had various unrelated clinical problems, including stroke, alcoholic shock, arteriopathy, peritonitis or myeloma. Two had diarrhoea and four subsequently died. The spirochaetes were isolated 
with a manual blood culture system and specialised media. Primary isolation of five isolates was achieved in Hémoline blood culture bottles (bioMérieux, Marcy L'Etoile, France) and the other in Bioargos Sanofi diagnostic anaerobic medium (Institut Pasteur, Paris, France) [16]. The isolate from the USA grew in the ESP automated blood culture system (Difco, Detroit, MI, USA), but failed to produce a signal [17]. Changes in the medium were noticed by an observant operator, who then subcultured from the bottle.

Spirochaetaemia associated with $B$. pilosicoli has not been reported in Australia despite the existence of groups of individuals known to have a high rate of intestinal carriage and who have other debilitating conditions which might predispose to bacterial translocation across the intestinal wall. These groups include Aboriginal people living in remote communities, recent migrants from developing countries, homosexual males and patients with HIV infection. Automated blood culture systems are now used routinely in most large hospital diagnostic laboratories in Australia, and it was hypothesised that these systems may fail to detect spirochaetaemia caused by $B$. pilosicoli. The main automated blood culture systems currently used are the BACTEC (Becton Dickinson Diagnostic Instrument Systems, Sparks, MD, USA) and BacT/Alert (Organon Teknika Corporation, Durham, NC, USA) systems; therefore, these were investigated. Both are fully automated, non-radiometric and use continuous, noninvasive monitoring of $\mathrm{CO}_{2}$ to detect microbial growth [18]. The BACTEC system uses a fluorescence sensor located in the medium [19]. The BacT/Alert system uses a colorimetric method of detection; when growth of the micro-organism produces $\mathrm{CO}_{2}$, the colour of the sensor changes from green to yellow [20].

Two manual systems were also investigated. Two types of manual Hémoline blood culture bottles were evaluated, as they were used to isolate B. pilosicoli from the blood of some of the original French patients. Manual BBL Septi-Chek blood culture bottles (Becton Dickinson Microbiology Systems, Cockeysville, MD, USA) with three different types of media were also tested, as these are often used to culture blood obtained from Australian Aboriginal patients in remote rural communities.

\section{Materials and methods}

\section{B. pilosicoli strains}

The nine strains of $B$. pilosicoli studied were obtained from the Reference Centre for Intestinal Spirochaetes at Murdoch University. They comprised five strains from blood (French strains 382/91, PE90, 28/94 and RA87, and the USA strain H1), three from human faeces (H171, WesB and H121) and the porcine type strain $\mathrm{P} 43 / 6 / 78^{\mathrm{T}}$ (ATCC $51139^{\mathrm{T}}$ ). The frozen $\left(-70^{\circ} \mathrm{C}\right.$ ) strains were thawed and inoculated into a pre-reduced anaerobic broth medium as described by Kunkle et al. [21] and checked for purity by phase contrast microscopy and culture on Columbia agar base containing horse blood 5\% (BA). Plates were incubated in an anaerobic chamber (Don Whitley Scientific, Shipley, Yorkshire) in an atmosphere of $\mathrm{N}_{2} 80 \%, \mathrm{H}_{2}$ $10 \%, \mathrm{CO}_{2} 10 \%$ at $37^{\circ} \mathrm{C}$ for 5 days. The spirochaetes grew as a thin haze with weak, incomplete haemolysis.

\section{Preparation of inocula}

The isolates were grown to log phase in the prereduced anaerobic broth, diluted in phosphate-buffered saline (PBS), $\mathrm{pH}$ 7.2, and counted with a Helber counting chamber, as $B$. pilosicoli does not form discrete countable colonies. Each suspension was adjusted to $1 \times 10^{5}$ organisms $/ \mathrm{ml}$ in PBS, then serial 10-fold dilutions were made in PBS to obtain concentrations of $10^{4}, 10^{3}$ and $10^{2}$ organisms $/ \mathrm{ml}$. A $1-\mathrm{ml}$ volume of each suspension was added to $9 \mathrm{ml}$ of human blood (Australian Red Cross) to give final concentrations of $1 \times 10^{4}, 1 \times 10^{3}, 1 \times 10^{2}$ and $1 \times$ $10^{1}$ organisms $/ \mathrm{ml}$ of blood. Blood comprised outdated RBCs from which plasma had been removed and the cells reconstituted with $100 \mathrm{ml}$ of Adsol (Baxter), consisting of dextrose, $\mathrm{NaCl}$, adenine and mannitol. The 10-ml volumes were used to inoculate the proprietary media. The viability of each strain was determined immediately after inoculation by subculture from the bottle with the highest spirochaete concentration on to $\mathrm{BA}$, followed by anaerobic incubation at $37^{\circ} \mathrm{C}$ for 5 days.

\section{Blood culture systems and media}

A summary of the blood culture systems and media used, and number of strains examined in each is presented in Table 1. Three strains of B. pilosicoli (382/91 and PE90 from blood, H171 from faeces) were tested in the manual Hémoline system, with both a trypticase soy agar slope in trypticase soy broth (diphasic) and a modified Wilkens Chalgren broth (anaerobic), and in the manual BBL Septi-Chek system with trypticase soy broth (TSB), modified brain heart infusion broth (BHIB) and thioglycollate broth bottles. These three strains were also tested separately in the two automated systems with BACTEC PLUS Anaero$\mathrm{bic} / \mathrm{F}$ with and without Fastidious Organism Supplement (FOS), and BacT/Alert Anaerobic and FAN Anaerobic culture bottles. The other six strains, 28/ 94, RA87, H1, WesB, H121 and P43/6/78 ${ }^{\mathrm{T}}$, were tested at the same time, but only in the BACTEC 9240 system with PLUS Anaerobic/F culture vials and in the BacT/Alert system with FAN Anaerobic culture bottles.

\section{Detection of spirochaete growth}

All bottles were incubated for up to 28 days. For the manual bottles, a $0.5-\mathrm{ml}$ sample was taken on days 2 , $5,7,10,14,18,21,24$ and 28 and plated on to BA 
Table 1. Blood culture systems and media tested

\begin{tabular}{llc}
\hline $\begin{array}{l}\text { Blood culture } \\
\text { system }\end{array}$ & Media & $\begin{array}{c}\text { Number of } \\
\text { strains tested }\end{array}$ \\
\hline Hémoline & Diphasic & $3^{*}$ \\
& Anaerobic & 3 \\
BBL Septi-Chek & TSB & 3 \\
& BHIB & 3 \\
BACTEC 9240 & Thioglycollate broth & 3 \\
& PLUS Anaerobic/F with FOS & 3 \\
BacT/Alert & FAUS Anaerobic/F Anaerobic & $3+6^{\dagger}$ \\
& Anaerobic & 3 \\
\end{tabular}

TSB, trypticase soy broth; BHIB, modified brain heart infusion broth; FOS, fastidious organism supplement.

${ }^{*}$ The three strains tested throughout were 382/91, PE90 and H171. ${ }^{\dagger}$ The other six strains wer 28/94, RA87, H1, WesB, H121 and P43/ $6 / 78^{\mathrm{T}}$

which was incubated anaerobically for up to 14 days. Where there was a suspicion of microbial growth at the time of sampling because of a colour change or build up of gas, a sample was also examined by phase contrast microscopy. For the manual bottles, the endpoint was defined as the day on which a sample was taken which subsequently yielded a growth of spirochaete on BA. Actual times between inoculation and confirmation of a positive result were longer than this because of the need for prolonged incubation of the plates.

For the automated systems, time to positivity was defined as the time of initial incubation until an automatic signal was detected or until a positive subculture was obtained. For bottles that did not give a signal, 0.5-ml samples were taken at 7, 14, 21 and 28 days and plated on to BA which was incubated anaerobically for up to 14 days. Care was taken not to disturb any gas present in the bottles when samples were removed. Where positive signals were obtained, the bottles were removed and a sample of broth was examined by phase contrast microscopy and plated on to BA. Spirochaete numbers in the broth were estimated with the counting chamber, after centrifugation at $150 \mathrm{~g}$ for $5 \mathrm{~min}$ to remove red blood cells.

\section{Results}

Growth of strains 382/91, PE90 and H171 in all systems

All three strains were viable when inoculated. Strain H171 was not detected in the manual BBL Septi-Chek system with thioglycollate broth, and none of the strains was detected by signal or subculture in the BacT/Alert system with FAN Anaerobic medium (Table 2). All three strains grew in the BacT/Alert Anaerobic medium, although strain 382/91 failed to produce a signal and was detected only by subculture. All the other systems and media supported growth of the spirochaete strains, but at various limits of detection and times to detection (Table 2). Strain 382/91 tended to be detected earlier and from a lower initial inoculum than the other two strains. Overall results were similar for a given strain for different manual systems or media with detection limits of between $10^{1}$ and $10^{4}$ spirochaetes $/ \mathrm{ml}$ of blood in the original inoculum. Individual manual bottles sampled between 2 and 10 days were positive by subculture and the plates generally took 5-8 days to show growth. In most cases, spirochaetes were recovered by subculture before they were detected by phase contrast microscopy.

\section{Growth of another six strains in automated systems}

Strains 28/94, RA87, H1, WesB, H121 and P43/6/78 were all viable, and grew and produced signals in the BACTEC system with PLUS Anaerobic/F without FOS (Table 2). Time to first detection by signal in this system for the six strains ranged from 5.7 to 14.9 days (mean 7.5 days) (Table 3) although, unexpectedly,

Table 2. Limits of detection and minimum time to detection at any inoculum concentration for B. pilosicoli strains 382/92, PE90 and H171 in manual and automated blood culture systems

\begin{tabular}{|c|c|c|c|}
\hline \multirow[b]{2}{*}{ Systems and media } & \multicolumn{3}{|c|}{$\begin{array}{l}\text { Lowest inoculum detected (minimum time to } \\
\text { detectable growth in days) }\end{array}$} \\
\hline & strain $382 / 91$ & strain PE90 & strain $\mathrm{H} 171$ \\
\hline Hémoline Diphasic & $10^{1}(2)$ & $10^{3}(5)$ & $10^{4}(7)$ \\
\hline Hémoline Anaerobic & $10^{2}(2)$ & $10^{2}(2)$ & $10^{4}(10)$ \\
\hline BBL Septi-Chek TSB & $10^{1}(2)$ & $10^{2}(2)$ & $10^{1}(7)$ \\
\hline BBL Septi-Chek BHIB & $10^{1}(2)$ & $10^{2}(5)$ & $10^{2}(7)$ \\
\hline BBL Septi-Chek Thioglycollate Broth & $10^{1}(2)$ & $10^{1}(5)$ & - \\
\hline BACTEC Plus Anaerobic/F & $10^{1}(5.6)^{*}$ & $10^{2}(10.7)^{*}$ & $10^{3}(7.3)^{*}$ \\
\hline BACTEC Plus Anaerobic/F FOS ${ }^{\S}$ & $10^{2}(6.7)^{*}$ & $10^{2}(9.1)^{*}$ & $10^{3}(7.3)^{*}$ \\
\hline BacT/Alert FAN Anaerobic & - & - & - \\
\hline BacT/Alert Anaerobic $^{\dagger}$ & $10^{4}(7)$ & $10^{3}(14.1)^{*}$ & $10^{3}(9.5)^{*}$ \\
\hline
\end{tabular}


Table 3. Limits of detection and time to detection for $B$. pilosicoli strains 28/94, RA87, H1, WesB, H121 and $\mathrm{P} 43 / 6 / 78^{\mathrm{T}}$ tested in automated systems

\begin{tabular}{lcc}
\hline & $\begin{array}{c}\text { Lowest inoculum detected (minimum time to } \\
\text { detection in days) }\end{array}$ \\
\cline { 2 - 3 } Strain no. & $\begin{array}{c}\text { BACTEC PLUS } \\
\text { Anaerobic/F }\end{array}$ & $\begin{array}{c}\text { BacT/Alert FAN } \\
\text { Anaerobic }\end{array}$ \\
\hline $28 / 94$ & $10^{2}(7.4)$ & - \\
RA87 & $10^{2}(8.3)$ & $10^{4}(14)$ \\
H1 & $10^{3}(14.9)$ & - \\
WesB & $10^{1}(5.7)$ & $10^{3}(7)$ \\
H121 & $10^{1}(8.5)$ & $10^{4}(7)$ \\
P43/6/78 & $10^{1}(6.9)$ & $10^{1}(7)$ \\
\hline
\end{tabular}

- , no growth detected.

* Detection by automated signal.

${ }^{\dagger}$ Detection by subculture, not signal. Subcultures were only taken on days $7,14,21$ and 28 and required incubation for a futher 5-8 days before detection was achieved.

strain $\mathrm{H} 1$ took 16.6 days to give a signal at the highest inoculum. Spirochaetes could be seen in wet mount preparations at the time of signalling. Generally, bottles containing the highest inoculum of a given strain signalled first. Limits of detection for the six strains varied between $10^{1}$ (strains WesB, H121 and P43/6/ $78^{\mathrm{T}}$ ) and $10^{3}$ (strain H1) spirochaetes $/ \mathrm{ml}$ of blood in the original inoculum. Positive bottles contained between $4 \times 10^{7}$ and $5 \times 10^{8}$ organisms $/ \mathrm{ml}$, confirming that the strains had proliferated. B. pilosicoli was subsequently detected by subculture from all bottles that signalled positive, in the subculture taken immediately before signalling. Growth from subculture took 2-8 days.

None of the bottles signalled positive in the BacT/Alert system with the FAN Anaerobic medium. However, four of the six strains were detected by subculture, P43/ $6 / 78^{\mathrm{T}}$ at all concentrations, WesB at $1 \times 10^{4}$ and $1 \times 10^{3}$, and H121 and RA87 at $1 \times 10^{4}$ spirochaetes/ $\mathrm{ml}$ of blood. Time to first detectable growth varied between 7 days for strains WesB, H121 and P43/6/78 and 14 days for strain RA87 (Table 3). Except for faecal isolate $\mathrm{H} 121$ at the $1 \times 10^{4}$ concentration, viable organisms were observed by microscopy and recovered by subculture from the vials at successive sampling points. A considerable build up of gas at 21 and 28 days was noticed in these vials. The graph of $\mathrm{CO}_{2}$ production over time produced by the BacT/Alert computer had a positive slope, indicating $\mathrm{CO}_{2}$ production in the vials, and the bases of the bottles also became yellow, but nevertheless they did not generate a signal.

\section{Discussion}

This study demonstrated that blood culture systems and protocols in common use in Australian diagnostic laboratories are suboptimal for detection of $B$. pilosicoli.
Studies of automated and manual systems in a clinical setting have shown that the majority of organisms encountered in blood require $<7$ days for detection. The 5-day retention of blood culture samples is regarded as a working compromise between maximum detection and cost-effectiveness [19, 22-24]. The present study held samples for 28 days so as to evaluate the recovery times of the slow-growing $B$. pilosicoli. As few as $1 \times 10^{1}$ spirochaetes $/ \mathrm{ml}$ of blood were detected in some cases (Tables 2 and 3). Although it is not known how many organisms are present in natural spirochaetaemias with $B$. pilosicoli, they have been isolated in patients with Hémoline media; in the present study these media had limits of detection of between $10^{1}$ and $10^{4}$ organisms $/ \mathrm{ml}$ of blood (Table 2). Overall, there was considerable variation in detection thresholds between the various strains tested, but the basis of this variability was not investigated. No consistent differences in growth were found between strains of faecal or blood origin.

Of the two automated systems tested, the BACTEC system performed best, with all $B$. pilosicoli strains growing and being detected by signal in the media. Even with the BACTEC system, the time required for detection considerably exceeded the 5-day retention normally used by clinical diagnostic laboratories. Consequently, spirochaetaemias with $B$. pilosicoli would not usually be detected. Addition of FOS to the media did not improve detection time. Besides enhancing the growth of fastidious organisms, FOS has a neutralising effect on sodium polyethol sulphonate (SPS), which is known to affect blood culture yield [25]. SPS is an anticoagulant agent that is also used to counteract the bactericidal activity of serum. The results suggest that $B$. pilosicoli is not susceptible to SPS at the concentration used in these media.

The BacT/Alert system performed poorly for the detection and recovery of B. pilosicoli. Strains 382/ 91, PE90 and H171 grew in the Anaerobic medium but not in the FAN Anaerobic medium. FAN Anaerobic medium contains Ecosorb, a proprietary material composed of activated carbon particles and Fuller's earth which is thought to non-specifically bind inhibitory factors other than antimicrobial agents [23]. Whilst designed to increase organism recovery, Ecosorb may have bound necessary growth requirements for $B$. pilosicoli, such as cholesterol, which is essential for growth of the closely related intestinal spirochaete $B$. hyodysenteriae [26]. Even with the Anaerobic medium, one of three strains did not generate a signal and time to detection was longer than with the BACTEC system. $B$. pilosicoli was only recovered from bottles inoculated with $10^{3}$ or $10^{4}$ spirochaetes $/ \mathrm{ml}$ of blood, which suggests that it was not optimal for growth of $B$. pilosicoli. Unfortunately, the other six strains were tested only in the FAN Anaerobic medium, and only one strain was detected in a bottle inoculated with $10^{1}$ spirochaetes $/ \mathrm{ml}$ of blood. It is possible that if the 
Anaerobic medium had been tested more strains may have grown and may have been more rapidly detected at lower concentrations.

Detection algorithms also contributed to the poor performance of the BacT/Alert system. The system did not signal the presence of $B$. pilosicoli even when a build up of $\mathrm{CO}_{2}$ had been generated and the organism had been recovered by subculture ( 4 of 9 strains; 8 of 36 bottles). It is unlikely that an algorithm would be changed to accommodate slow-growing organisms such as B. pilosicoli.

Although they are not widely used in Australia, growth in the two manual Hémoline preparations confirmed the utility of these media for isolation of $B$. pilosicoli from blood [16]. The manual Septi-Chek bottles gave comparable results. Therefore, either Hémoline or Septi-Chek could be used with some confidence to detect $B$. pilosicoli spirochaetaemia in laboratories where automated systems are not available, provided that the vials were incubated for a longer time to accommodate the organism's slow growth. The manual bottles required considerable time and labour to monitor and, in particular needed regular blind subculturing to detect the organism. While minimum time to positivity by subculture varied from 2 to 10 days, which was earlier than for the BACTEC system, the organisms were often not at a high enough concentration in the bottles to be visible by microscopy. Also, subcultures on plates needed to be incubated anaerobically for 5-8 days before growth was detected.

This study indicates that the BACTEC system is better than the BacT/Alert system for the detection of $B$. pilosicoli in seeded blood culture and, hence, is likely to be superior for the detection of spirochaetaemias in patients. Unfortunately an 18-day incubation period with the BACTEC PLUS Anaerobic/F Culture Vials would be needed to detect the majority of clinical isolates. If the BacT/Alert system is used then BacT/ Alert Anaerobic Culture Bottles should be considered and terminal subculturing performed. Both manual bottles tested required a minimum of an 18-day protocol with blind subculture every 3 days to enable early detection of viable strains. In addition, an aspirate from any positive bottle should be examined by phase contrast microscopy, as B. pilosicoli is slender and very difficult to detect by Gram's stain. Subcultures on BA should be incubated anaerobically for up to 10 days before being discarded.

Spirochaetaemia associated with B. pilosicoli should be considered in febrile patients from groups known to have a high rate of intestinal carriage with the organism. Appropriate media and conditions, including prolonged incubation times, are required to detect these organisms. Prospective studies to determine how frequently spirochaetaemias associated with B. pilosi- coli occur as a clinical complication in these high-risk groups of patients should be initiated.

This study was supported by a grant from the National Health and Medical Research Council of Australia. The assistance of staff of the Departments of Microbiology and Infectious Diseases at the Western Australian Centre of Pathology and Medical Research and Royal Perth Hospital, particularly Leigh Mulgrave and Geoff Coombs, is gratefully acknowledged. Sophy Oxberry kindly provided spirochaete cultures, whilst Becton Dickinson, Organon Teknika and bioMérieux generously donated some of the media used.

\section{References}

1. Lee JI, Hampson DJ. Genetic characterisation of intestinal spirochaetes and their association with disease. $J$ Med Microbiol 1994; 40: 365-371.

2. Trott DJ, Combs BG, Mikosza ASJ et al. The prevalence of Serpulina pilosicoli in humans and domestic animals in the Eastern Highlands of Papua New Guinea. Epidemiol Infect 1997; 119: 369-379.

3 Trott DJ, Stanton TB, Jensen NS, Duhamel GE, Johnson JL, Hampson DJ. Serpulina pilosicoli sp. nov., the agent of porcine intestinal spirochetosis. Int J Syst Bacteriol 1996; 46: 206-215.

4. Taylor DJ, Simmons JR, Laird HM. Production of diarrhoea and dysentery in pigs by feeding pure cultures of a spirochaete differing from Treponema hyodysenteriae. Vet Rec 1980; 106: $326-332$.

5. Trivett-Moore NL, Gilbert GL, Law CLH, Trott DJ, Hampson DJ. Isolation of Serpulina pilosicoli from rectal biopsy specimens showing evidence of intestinal spirochetosis. J Clin Microbiol 1998; 36: 261-265.

6. Barrett SP. Intestinal spirochaetes in a Gulf Arab population. Epidemiol Infect 1990; 104: 261-266.

7. Lee JI, Hampson DJ. Intestinal spirochaetes colonizing Aborigines from communities in the remote north of Western Australia. Epidemiol Infect 1992; 109: 133-141.

8. Käsbohrer A, Gelderblom HR, Arasteh $\mathrm{K}$ et al. Intestinale spirochätose bei HIV-infektion. Vorkommen, Isolierung und Morphologie der Spirochäten. Dtsch Med Woschenschr 1990; 115: $1499-1506$.

9. Gad A, Willén R, Furugård K, Fors B, Hradsky M. Intestinal spirochaetosis as a cause of longstanding diarrhoea. Ups J Med Sci 1977; 82: 49-54.

10. Douglas JG, Crucioli V. Spirochaetosis: a remediable cause of diarrhoea and rectal bleeding? BMJ 1981; 283: 1362.

11. Oxberry SL, Trott DJ, Hampson DJ. Serpulina pilosicoli, waterbirds and water: potential sources of infection for humans and other animals. Epidemiol Infect 1998; 121: 219-225.

12. Kostman JR, Patel M, Catalano E, Camacho J, Hoffpauir J, DiNubile MJ. Invasive colitis and hepatitis due to previously uncharacterized spirochetes in patients with advanced human immumodeficiency virus infection. Clin Infect Dis 1995; 21: 1159-1165.

13. Padmanabhan V, Dahlstrom J, Maxwell L, Kaye G, Clarke A, Barratt PJ. Invasive intestinal spirochetosis: a report of three cases. Pathology 1996; 28: 283-286.

14. Hovind-Hougen K, Birch-Andersen A, Henrik-Nielsen R et al. Intestinal spirochetosis: Morphological characterization and cultivation of the spirochete Brachyspira aalborgi gen. nov., sp. nov. J Clin Microbiol 1982; 16: 1127-1136.

15 Mikosza ASJ, La T, Brooke CJ et al. PCR amplification from fixed tissue indicates frequent involvement of Brachyspira aalborgi in human intestinal spirochetosis. J Clin Microbiol 1999; 37: 2093-2098.

16. Fournié-Amazouz E, Baranton G, Carlier JP et al. Isolations of intestinal spirochaetes from the blood of human patients. $J$ Hosp Infect 1995; 30: 160-162.

17. Trott DJ, Jensen NS, Saint Girons I et al. Identification and characterization of Serpulina pilosicoli isolates recovered from the blood of critically ill patients. J Clin Microbiol 1997; 35: $482-485$.

18. Smith JA, Bryce EA, Ngui-Yen JH, Roberts FJ. Comparison of BACTEC 9240 and BacT/Alert blood culture systems in an adult hospital. J Clin Microbiol 1995; 33: 1905-1908.

19. Nolte FS, Williams JM, Jerris RC et al. Multicenter clinical evaluation of a continuous monitoring blood culture system 
using fluorescent-sensor technology (BACTEC 9240). J Clin Microbiol 1993; 31: 552-557.

20. Thorpe TC, Wilson ML, Turner JE et al. BacT/Alert: an automated colorimetric microbial detection system. J Clin Microbiol 1990; 28: 1608-1612.

21. Kunkle RA, Harris DL, Kinyon JM. Autoclaved liquid medium for propagation of Treponema hyodysenteriae. J Clin Microbiol 1986; 24: 669-671.

22. Hardy DJ, Hulbert BB, Migneault PC. Time to detection of positive BacT/Alert blood cultures and lack of need for routine subculture of 5- to 7-day negative cultures. J Clin Microbiol 1992; 30: 2743-2745.

23. Wilson ML, Weinstein MP, Mirrett $\mathrm{S}$ et al. Controlled evaluation of BacT/Alert Standard anearobic and FAN anaerobic blood culture bottles for the detection of bacteremia and fungemia. $J$ Clin Microbiol 1995; 33: 2265-2270.

24. Doern GV, Davaro R, George M, Campognone P. Lack of requirement for prolonged incubation of Septi-Chek blood culture bottles in patients with bacteremia due to fastidious bacteria. Diagn Microbiol Infect Dis 1996; 24: 141-143.

25. Eng J. Effect of sodium polyanethol sulfonate in blood cultures. J Clin Microbiol 1975; 1: 119-123.

26. Stanton TB, Cornell CP. Erythrocytes as a source of essential lipids for Treponema hyodysenteriae. Infect Immun 1987; 55: 304-308. 